\title{
Criação de personagens lúdicos para capacitação em serviço da equipe de
}

\section{Enfermagem sobre anticoagulantes orais}

\author{
Creation of playful characters for in-service training of the Nursing staff on oral anticoagulants \\ Creación de personajes lúdicos para la formación en servicio del equipo de Enfermería en
}

anticoagulantes orales

Recebido: 24/02/2021 | Revisado: 03/03/2021 | Aceito: 07/03/2021 | Publicado: 15/03/2021

\author{
Adriana da Costa Coelho \\ ORCID: https://orcid.org/0000-0003-2047-9592 \\ Universidade Federal do Estado do Rio de Janeiro, Brasil \\ E-mail: dricoelho10@hotmail.com \\ Dasymar Martins da Silva Lucas \\ ORCID: https://orcid.org/0000-0002-6380-6031 \\ Universidade Federal do Estado do Rio de Janeiro, Brasil \\ E-mail: dasymar.lucas@gmail.com \\ Geovane de Kassio Nunes \\ ORCID: https://orcid.org/0000-0002-8446-3103 \\ Universidade Federal do Estado do Rio de Janeiro, Brasil \\ E-mail: giodekassio@gmail.com \\ Milena Preissler das Neves \\ ORCID: https://orcid.org/0000-0002-3890-924X \\ Universidade Veiga de Almeida, Brasil \\ E-mail: milenapreissler@gmail.com \\ Leandro Andrade da Silva \\ ORCID: https://orcid.org/0000-0003-3213-5527 \\ Universidade Veiga de Almeida, Brasil \\ E-mail: proflandrade@gmail.com \\ Teresa Tonini \\ ORCID: https://orcid.org/0000-0002-5253-2485 \\ Universidade Federal do Estado do Rio de Janeiro, Brasil \\ E-mail: ttonini@terra.com.br \\ Renata Flavia Abreu da Silva \\ ORCID: https://orcid.org/0000-0003-1776-021X \\ Universidade Federal do Estado do Rio de Janeiro, Brasil \\ E-mail: renata.f.silva@unirio.br
}

\begin{abstract}
Resumo
A criação de personagens lúdicos para capacitação em serviço da equipe de Enfermagem sobre anticoagulantes orais propõe-se a promover estratégias para treinamento que contribuam na superação de lacunas entre o saber e o fazer quanto às vulnerabilidades dos riscos da terapia de anticoagulação. Objetivo: descrever a criação e registro de personagens lúdicos para serem utilizados como instrumentos facilitadores na aprendizagem da equipe de Enfermagem quanto aos cuidados na administração dos anticoagulantes orais. Método: Estudo metodológico de abordagem qualitativa, a partir do desenvolvimento de dois personagens fictícios nomeados de "ACO e NACO". Resultados: Os personagens fictícios, criados para serem agentes facilitadores da aprendizagem, podem ser reconhecidos como instrumento pedagógico, lúdico e importante para reflexão da equipe de Enfermagem na administração dos anticoagulantes orais. O cenário hospitalar é um ambiente complexo, por possuir estruturas de gestão bastante rígidas, repetitivas e pré-estabelecidas. Ao mesmo passo, exige-se por parte da equipe de Enfermagem a busca da superação de lacunas entre o conhecimento teórico e a prática. Considerações Finais: Os personagens sugerem uma forma de aproximação e motivação da Enfermagem em buscar mais conhecimentos sobre anticoagulantes orais, fundamentando-se na linguagem, pensamento crítico e imaginação.
\end{abstract}

Palavras-chave: Anticoagulantes; Capacitação em serviço; Equipe de enfermagem; Asssistência ao paciente.

\begin{abstract}
The creation of playful characters for in-service training of the Nursing team on oral anticoagulants aims to promote training strategies that contribute to overcoming gaps between knowledge and action regarding the vulnerabilities of anticoagulation therapy risks. Objective: to describe the creation and registration of playful characters to be used as facilitating instruments in the learning of the Nursing team regarding care in the administration of oral anticoagulants. Method: Methodological study with a qualitative approach, based on the development of two fictional characters
\end{abstract}


named "ACO and NACO". Results: The fictional characters, created to be agents that facilitate learning, can be recognized as a pedagogical, playful, and important tool for reflection by the Nursing team in the administration of oral anticoagulants. The hospital scenario is a complex environment, as it has very rigid, repetitive, and preestablished management structures. At the same step, the nursing team is required to seek to bridge gaps between theoretical knowledge and practice. Final Considerations: The characters suggest a way of approaching and motivating Nursing to seek more knowledge about oral anticoagulants, based on language, critical thinking and imagination.

Keywords: Anticoagulants; Inservice training; Nursing team; Patient care.

\section{Resumen}

La creación de personajes lúdicos para la formación en servicio del equipo de Enfermería en anticoagulantes orales tiene como objetivo promover estrategias de formación que contribuyan a superar las brechas entre el conocimiento y la acción sobre las vulnerabilidades de los riesgos de la terapia anticoagulante. Objetivo: describir la creación y registro de personajes lúdicos para ser utilizados como instrumentos facilitadores en el aprendizaje del equipo de Enfermería sobre el cuidado en la administración de anticoagulantes orales. Método: Estudio metodológico con enfoque cualitativo, basado en el desarrollo de dos personajes de ficción denominados "ACO y NACO". Resultados: Los personajes de ficción, creados para ser agentes facilitadores del aprendizaje, pueden ser reconocidos como una herramienta pedagógica, lúdica e importante de reflexión por parte del equipo de Enfermería en la administración de anticoagulantes orales. El escenario hospitalario es un entorno complejo, ya que cuenta con estructuras de gestión muy rígidas, repetitivas y preestablecidas. En el mismo paso, se requiere que el equipo de enfermería busque cerrar las brechas entre el conocimiento teórico y la práctica. Consideraciones finales: Los personajes sugieren una forma de abordar y motivar a la Enfermería para buscar un mayor conocimiento sobre los anticoagulantes orales, basado en el lenguaje, el pensamiento crítico y la imaginación.

Palabras clave: Anticoagulantes; Capacitación en servicio; Grupo de enfermería; Atención al paciente.

\section{Introdução}

Os anticoagulantes orais são fármacos utilizados com intuito de manter níveis adequados da coagulação do sangue, interferindo na hemostasia (Serrano Júnior et al. 2019). Estes são prescritos para os pacientes portadores de doenças cardiovasculares tais como a fibrilação atrial e doenças valvulares. A administração desses medicamentos requer manejo rigoroso de cuidados da equipe de Enfermagem, devido à janela terapêutica ser bastante estreita (Costa et al. 2018).

Para manejo da administração dessa terapia de anticoagulação, se exige um saber-fazer pautado nos conhecimentos técnico-científicos de gerais e específicos relacionados às competências comunicacionais e atitudinais na relação profissionalcliente e profissional-profissional, em prol da segurança do paciente.

O Código de Ética dos Profissionais de Enfermagem, publicado pela Resolução COFEN N 0564/2017, assegura o direito dos exercentes de Enfermagem ao aprimoramento de conhecimentos científicos e técnicos (Freitas et. al. 2017). Assim, a Capacitação em Serviço é imperiosa para o fortalecimento do alicerce da qualidade dos cuidados de enfermagem e a incorporação de ferramentas assistenciais na tomada de decisão clínica dos enfermeiros.

Buscar métodos e estratégias eficazes e mais contributivos se torna fundamental para promoção na capacitação dos profissionais no cenário hospitalar, de modo que tenham melhores condições para repensar a administração de medicamentos, busquem a melhoria na qualidade e segurança no procedimento realizado (Simonette, Massa \& França 2017). A Capacitação/Treinamento em Serviço na Educação Profissional e Tecnológica requer uma aprendizagem significativa, contextualizada, orientada para o uso das Tecnologias da Informação e Comunicação, favorecendo o uso intensivo dos recursos da inteligência, que gere habilidades em resolver problemas e conduzir projetos nos diversos segmentos do setor produtivo. Como contraponto, pode-se afirmar que este tipo de aprendizagem deve estar cada vez mais distante da aprendizagem convencional, fundamentada no poder do verbo, teórica e dependente do uso intensivo da memória (Lima, 2016).

Dada à magnitude e vulnerabilidade dos riscos da terapia de anticoagulação e a capacitação dos profissionais de enfermagem, se questiona: Como promover estratégias para treinamento em serviço que de fato possam contribuir na superação de lacunas entre o saber e o fazer? 
As reflexões apresentadas propiciaram a ideia de criação de dois personagens fictícios nomeados: "ACO e NACO". Estes personagens lúdicos foram desenvolvidos com a finalidade de contribuir na reflexão do que fazer, se foi feito o que se pensou e no pensar sobre o que já se fez para consolidação do conhecimento sobre os cuidados de enfermagem na administração dos anticoagulantes orais durante a internação do paciente.

Os personagens foram construídos como material didático exclusivo para facilitar a retenção das informações repassadas e transformar os treinamentos em uma jornada criativa, instigante e inquietante.

Com mudanças proporcionadas pela era digital, os profissionais mudaram a forma de buscar informações e os métodos tradicionais de ensino tendem a se tornar desmotivantes. Por seu forte caráter motivacional, os personagens lúdicos têm sido usados como uma ferramenta alternativa para aprendizado formal (Lima, 2016).

As atividades lúdicas no processo de aprendizagem permitem que o receptor da mensagem interaja com o assunto através da audição, discussão, questionamentos, incentivando a produção do saber, rompendo assim a barreira criada de que o emissor é a única fonte de informação e conhecimento (Lima, 2016).

Neste sentido, a criação e utilização de personagens no processo de educação dos profissionais de saúde, com ênfase na apreciação das metodologias ativas de ensino-aprendizagem é uma proposta de elaboração de ferramentas que colaborem para preencher lacunas na prática profissional.

Para Rocha, Giordani, Fujita e Reinaldi (2020), é importante que as instituições de Ensino Superior que ofertam cursos de graduação em Enfermagem, fortaleçam a capacitação pedagógica por meio de disciplinas voltadas ao ensino, incentivando o pensamento crítico, ativo e reflexivo sobre atividades educacionais utilizando metodologias ativas.

$\mathrm{O}$ uso de personagens específicos para atividade educacional da equipe de Enfermagem na administração dos anticoagulantes orais poderá ser capaz de desencadear uma visão do todo - de interdependência e autonomia do profissional e da segurança do paciente. $\mathrm{O}$ uso de metodologias ativas na aprendizagem possibilita aos alunos/profissionais assimilarem maior volume de conteúdo técnico, reterem com mais facilidade as informações, armazená-las por mais tempo, além de gerar maior prazer e satisfação das aulas (Freitas et al. 2017; Barbosa \& Moura 2013).

O estudo se justifica pelo fato de que novas metodologias podem ser relevantes na redução de possíveis falhas no processo de administração dos anticoagulantes orais, podendo reduzir eventos adversos, custos e tempo da internação e reinternações frequentes favorecendo a sobrevida desses pacientes. Desta forma pode servir de guia para novos trabalhos que exijam esta mesma atividade.

A pesquisa pode contribuir com a equipe de enfermagem, uma vez que a formação e a (re)construção dos conhecimentos dos profissionais de saúde tem sido pautadas no uso de metodologias conservadoras ou tradicionais pouco eficazes e efetivas. Desta forma, torna-se inevitável (re)discutir e (re)criar processos de ensino e aprendizagem para o trabalho em saúde. Pois, perpassa modificações que refletem um comportamento coerente com o mundo atual.

Portanto, o objetivo deste estudo é descrever a criação e registro de dois personagens lúdicos para serem utilizados como instrumentos facilitadores na aprendizagem da equipe de Enfermagem quanto aos cuidados na administração dos anticoagulantes orais.

\section{Metodologia}

Trata-se de um estudo metodológico de abordagem qualitativa, onde se necessita que o desenvolvimento de instrumentos seja adequado à realidade e compreendidos por um grupo, modificando ideias e conceitos preexistentes (Fakih, Freitas \& Secoli 2009). De forma sistemática, os conhecimentos são utilizados pelo pesquisador na elaboração de um instrumento confiável, preciso e pragmático, que possa ser empregado por outros pesquisadores (Polit \& Beck, 2018).

Para produção dos Personagens Lúdicos com vistas à capacitação em serviço da equipe de enfermagem sobre 
anticoagulantes orais, três etapas foram desenvolvidas, a saber: revisão integrativa; o processo para criação dos personagens e o processo de registro dos personagens.

Por ser um dos métodos disponíveis para incorporação de evidências na prática clínica, a revisão integrativa foi realizada com a finalidade de se fundamentar o tema e criação dos personagens NACO e ACO, quanto ao manejo dos cuidados de enfermagem na terapêutica com anticoagulantes orais diretos e antagonistas de vitamina $\mathrm{K}$, com o objetivo de conhecer os cuidados de Enfermagem relacionados ao uso de anticoagulantes orais e seu manejo clínico.

A Revisão integrativa teve como questão norteadora: Quais evidências disponíveis na literatura sobre os cuidados de enfermagem na administração dos anticoagulantes orais em pacientes adultos internados?

A Busca da amostragem bibliográfica foi feita nas bases de dados: CINAHL, LILACS, MEDLINE, BDENF e os descritores utilizados foram: anticoagulantes AND cuidados de enfermagem, anticoagulant AND nursing care, nursing care AND oral anticoagulant. Pesquisou-se artigos em inglês, francês, português e espanhol em um recorte temporal de 6 anos (2013-2019) devido a publicação da portaria No 529 de 2013 sobre segurança do paciente.

Como critérios de inclusão foram artigos que descrevessem cuidados específicos de enfermagem na administração de anticoagulantes orais. Os critérios de exclusão: artigos que abordassem anticoagulantes venosos ou subcutâneos e artigos repetidos. A busca resultou na seleção total de 1309 artigos dos quais 40 foram selecionados pela leitura dos títulos para leitura dos resumos. A partir da leitura dos resumos 33 artigos foram selecionados para leitura na integra e 13 foram escolhidos para compor esta pesquisa.

Com o tratamento dos dados emergiram três temas: Educação em saúde, farmacodinâmica e farmacocinética dos anticoagulantes orais e Cuidados de Enfermagem. Esses temas serviram de fundamentação para a criação intelectual dos personagens subdividida em dois momentos: desenvolvimento intelectual e elaboração dos personagens. Após a etapa de criação, seguiu-se a etapa de registro dos personagens.

Na criação de um personagem, o pesquisador deve adotar passos seguidos por autores/roteiristas de cinema, em que o feeling e estilo são fundamentais e estão longe de ser um processo automático; ou seja, é necessário dar um propósito de existência para o personagem. Esse primeiro momento é para dar asas à imaginação em busca da essência do personagem, sem se preocupar com dados mais concretos como nome, corpo, idade, indumentária que se constituem como o momento seguinte. O interesse na criação é desvelar a alma do personagem, para alinhavar os seus atributos: tímido ou extrovertido, infantil ou sério, engraçado ou sisudo, entre outros. Todo esse processo deve ser registrado pelo pesquisador para que não perca essas qualidades quando for desenhá-lo com suas características físicas (Field, 1995).

Além desse registro dos personagens, é fundamental se garantir propriedade intelectual da obra criada pelos pesquisadores, segundo as diretrizes e normativas da instituição a qual estão vinculados - neste caso, a Universidade Federal do Estado do Rio de Janeiro - e da Fundação Biblioteca Nacional.

Este trabalho cumpre as exigências estabelecidas na Resolução N510, de 07 de abril de 2016 que dispõe sobre as normas e métodos aplicáveis a pesquisas em Ciências Humanas e Sociais. A pesquisa realizada, exclusivamente, com textos científicos para revisão da literatura científica não é registrada e, tampouco, avaliada pelo sistema CEP/CONEP.

\section{Resultados}

Com base na Revisão Integrativa, houve o desenvolvimento das Etapas "a" criação e Etapa "b" registro dos personagens, discutidas neste artigo. 


\subsection{Etapa de criação}

\subsubsection{Primeiro momento: desenvolvimento intelectual}

Com o estudo dos artigos encontrados foi visto, que o fármaco do grupo dos anticoagulantes orais, a Varfarina é usada na prevenção das tromboses produzindo um efeito anticoagulante indireto ao interferir no metabolismo da vitamina $\mathrm{K}$, tem mais interações fármaco-alimentar e necessita de monitorizações contínuas do International Normalized Ratio (INR). As principais substâncias potencializadoras a resposta dessa droga e geradora do elevado risco de hemorragia são o álcool (grandes quantidades ou ingestão crônica) e o Ácido Acetil Salicílico, além de outras drogas como o clopidogrel, abciximab, andrógenos, fluconazol emetronidazol.

Nesse mesmo sentido, Barbosa et al. (2020) expuseram no seu estudo sobre a importância dos cuidados de prevenção primárias e secundárias do tromboembolismo e na presença de alguns problemas cardíacos, pois a utilização da Varfarina requer controle clínico rigoroso dos profissionais de saúde.

Pacientes com insuficiência hepática também possuem o efeito dessa medicação potencializado. A atividade desse anticoagulante pode ser inibida por contraceptivos orais do tipo estrógenos, a aminoglutetimida, a carbamazepina e outros. Assim como a colestiramina e o sulcralfato podem reduzir a resposta à anticoagulação promovida.

Esse conhecimento fundamentou a importância de a equipe de enfermagem verificar quais outros medicamentos o paciente faz em uso em conjunto ao de anticoagulante oral. Outro item enfatizado foi que devido ao seu tempo de meia vida prolongado, há um nível de segurança maior em caso de doses esquecidas. Os novos anticoagulantes orais diretos antagonistas de vitamina possuem ação iniciada de 24 a 72 horas, com pico máximo de 72 a 96 horas. A meia vida de 1-3 dias, com duração de 2-5 dias. Portanto, a equipe de enfermagem deve se atentar quanto ao uso em pacientes com Insuficiência cardíaca, falência hepática, hipertireoidismo, idosos, deficientes de vitamina $\mathrm{K}$, perda ou ganho de peso e mulheres que amamentam.

O cuidado com a dosagem do medicamento varia de acordo com a clínica do paciente. Em altas doses pode causar anemia, sangramento e hemorragia, dor no peito, hepatite, tontura, letargia, urticária, alopecia, epistaxe, entre outros. Todavia os anticoagulantes orais diretos são mais eficazes e fáceis de manipular, pois não requerem rigoroso monitoramento no INR e há menores riscos de hemorragias intracranianas fornecendo resultados satisfatórios em relação à profilaxia básica.

Um dos critérios para uso deste anticoagulante é apresentar ao cliente todas as vantagens e desvantagens do uso, orientando-os em relação ao que fazer com doses esquecidas e o fato de que, em pacientes anticoagulados, o paracetamol é o analgésico mais seguro disponível sem receita médica. Destaca-se a necessidade do estímulo ao autocuidado que se concentra em fornecer educação com o objetivo de permitir que os pacientes façam as mudanças necessárias para que se tornem gestores de sua própria condição de saúde.

Novos anticoagulantes (Dabigatrana Rivaroxabana, Apixaban e Edoxaban) surgiram bem depois dos anticoagulantes convencionais em virtude das limitações dos convencionais e tem como características farmacológicas o rápido início de ação farmacocinética, baixa interação com outros medicamentos e alimentos, aumento da janela terapêutica e o baixo risco potencial de complicações hemorrágicas. A investigação farmacológica desses novos anticoagulantes está focada no desdobramento de novas moléculas de trombina e fator Xa. Farmacodinâmica e farmacocinética previsível e consistente e que seja administrado por via oral. Conclui-se que, os antagonistas de vitamina $\mathrm{K}$ requerem cuidados de enfermagem específicos quanto ao manejo, administração e monitoramento além de rigorosa atenção quanto às interações farmacológicas e alimentares.

No que se refere aos anticoagulantes diretos é imprescindível que haja estudos abordando os cuidados de enfermagem, mesmo que a monitorização do INR não seja contínua e que se tenha menos interações farmacológicas. Há necessidade de conhecer os fatores facilitadores para a compreensão dos pacientes e profissionais sobre as questões relacionadas aos anticoagulantes, contribuintes às mudanças duradouras no comportamento. Desde modo, tanto a educação em saúde dos pacientes quanto a capacitação profissional devem ser inerentes ao cuidado de pacientes anticoagulados. Ainda há poucas 
evidências que os Enfermeiros aproveitam as oportunidades na administração de anticoagulantes orais para realizar a educação em saúde.

Ressalta-se que a equipe de Enfermagem é responsável pelo cuidado integral do paciente em uso de terapia de anticoagulação. Desde o recebimento dos medicamentos até o monitoramento da dosagem, efeitos adversos e promoção do autocuidado do paciente.

\subsubsection{Segundo momento: elaboração dos personagens}

Este momento foi acrescido de uma busca da história dos anticoagulantes orais sob os seguintes critérios de inclusão: abordagem histórica exclusiva dos anticoagulantes sem recorte temporal. Como resultado encontrou-se apenas 01 artigo.

O desenvolvimento dos personagens "ACO" e "NACO", de autoria intelectual de um dos pesquisadores, necessitou da ajuda de um profissional de design gráfico para execução da proposta. "ACO" foi baseado no medicamento mais recomendado pelas Diretrizes Brasileiras de anticoagulantes: Varfarina Sódica (Costa et al. 2018).

A elaboração do personagem "ACO" foi baseada no formato e cores da embalagem da Varfarina Sódica. O personagem apresenta feições bastante simpáticas e atrativas, uma camiseta com a identificação da abreviação de anticoagulante oral - ACO e aparência de um invólucro de medicamento. O personagem, segura uma placa análoga de trânsito com a escrita "stop", em que ressalta o cuidado e o receio que o profissional precisa ter para manusear tal medicação, além de estar alerta ao uso abusivo deste fármaco. A opção da Cor Vermelha decorre do fato de que esta cor era preferida dos soberanos e súditos das classes altas do século XVI; outros significados atribuídos à cor vermelha, ao personagem é soberania mercadológica há mais de 50 anos e a mais recomendada nas diretrizes brasileiras e mundiais de anticoagulantes (Costa et al. 2018).

A Varfarina sódica foi utilizada pela primeira vez como raticida em 1948. Assim se pensou na figura de um rato no verso da camiseta. Porém, a possível associação com o personagem Mickey Mouse®do Walt Disney seria conflituosa e perderia todo o sentido da mensagem que o personagem "ACO" pretende transmitir. Talvez repulsa por parte do público ao seu uso, dado aos sentimentos de medo/terror a provocação de nojo quanto ao grau de contaminação e agravos relacionados aos ratos (Mendes, Silveira \& Galvão 2008).

Posteriormente, pensou-se em colocar uma faixa na cabeça deste personagem com a seguinte escrita RNI. Esse pensamento ocorreu pelo fato de que um dos principais cuidados que a enfermagem deve ter ao administrar a Varfarina é o monitoramento do tempo da Razão Normalizada Internacional - RNI, que é o teste mais amplamente usado para controlar os níveis de anticoagulação oral. Optou-se por descartar a faixa, por acreditar que, esteticamente, poderia haver rejeição da imagem e canalizaria o foco da equipe de enfermagem apenas na monitorização de INR (Costa et al. 2018).

"NACO" foi baseado nos novos anticoagulantes (Dabigatrana Rivaroxabana, Apixaban e Edoxaban), estruturado a partir de características dos medicamentos anticoagulantes que o inspirou. Este personagem representa a terapêutica de anticoagulação oral em transformação com os recentes avanços farmacológicos para esse grupo de medicamento. Sua característica mais marcante é a jovialidade, modernidade e disposição para alcançar o "padrão ouro" na terapia de anticoagulação com o que há de tecnologia mais moderna disponível. A jovialidade pensada para o personagem "NACO" é devida a este grupo de medicamentos ter surgido bem depois dos anticoagulantes orais convencionais.

Este personagem possui um cordão de ouro bastante destacado. Este acessório foi desenhado na intenção de remeter a constante busca que o profissional de saúde deve ter pelo "padrão-ouro" dos procedimentos e terapêuticas utilizadas.

$\mathrm{O}$ uso destes personagens faz com que o profissional compreenda, imediatamente, a realidade através de imagem oferecida, comportamento e gestos. A simplicidade da estrutura de pensamento estabelece uma conexão direta com a equipe de enfermagem que está sendo capacitada. 


\subsection{Etapa de registro de obra intelectual}

Sequencialmente à criação dos personagens, iniciou-se o processo de registro sob a denominação de Família "ACO". A escolha de uma família no lugar de personagens individuais baseou-se em dois critérios: financeiro, já que o registro de uma família é menos oneroso, e na possibilidade de futuramente criar novos personagens "aumentando esta família".

Conforme alei nº610/98, foi necessário o comparecimento ao escritório de Direitos Autoral da Fundação Biblioteca Nacional que fica localizado na Avenida Presidente Vargas, 3131, sala 702, bairro da Cidade Nova, no estado do Rio de Janeiro, para preenchimento dos formulários de registro de direitos autorais.

Desde 1898, quando foi publicada a primeira lei específica sobre direitos autorais no Brasil, até hoje, a Biblioteca Nacional é responsável pela certificação pública de obras intelectuais para segurança jurídica dos direitos morais e patrimoniais do autor. Vale ressaltar que o registro se refere às criações de formas e não as ideias.

Para esta solicitação, foi necessário preencher um formulário, preparar uma cópia física da obra intelectual em folhas avulsas de papel A4 e pagar um Guia de Recolhimento da União (GRU) - A Lei do Direito Autoral (9.610/98) cessou a gratuidade do registro.

O passo a passo para o registro de obra intelectual pode ser obtido através do endereço eletrônico https://www.bn.gov.br/servicos/direitos-autorais.

Em maio de 2019 a documentação foi conferida e protocolada no momento do recebimento e emitido um comprovante de protocolo com número identificador do processo. Em 23/01/2019 foi informado que a obra foi registrada sob o $\mathrm{n}^{\circ} 812.649$ conforme os seguintes dados: Família ACO. Obra registrada com número de registro 812.649. A certidão reproduz a imagem da página 1 da obra. Protocolo 2019RJ_6471, em 08/05/2019. Registro em 23/01/2020, L.1580 F.201. Personagem/desenho/fotografia, 2 página(s). Inédita.

Figura 1 - Representação ilustrativa da família “ACO”: Personagens ACO e NACO. Rio de Janeiro, Brasil. 2019.
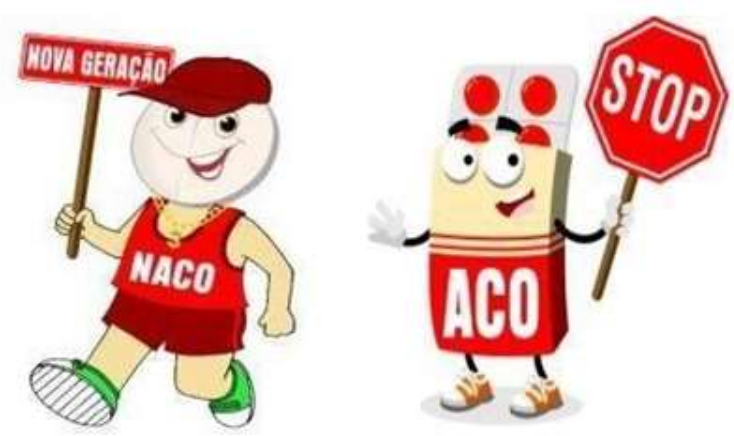

Fonte: Autores.

\section{Discussão}

A comunicação é utilizada pelo homem para se expressar e propagar ideias. O uso de imagens é uma forma de comunicação não verbal mais utilizada pela civilização humana. Este tipo de comunicação com imagens, figuras, ou pinturas associadas a determinado assunto, pode ser denominada de iconografia (Mendes 2008).

O conceito de iconografia pode ser definido como o estudo descritivo da representação visual de símbolos e imagens (Mendes 2008). Optou-se por este processo de criação de personagens pelo fato de envolver os indivíduos, e despertar uma vontade de aprender. Trata-se é uma forma de aprendizado ativo no plano imaginário, com possibilidade de aumentar o envolvimento da equipe e sua corresponsabilização no processo de cuidados de saúde, promovendo a melhoria da qualidade dos processos, a satisfação dos pacientes e motivação da equipe de Enfermagem. Portanto, torna-se o envolvimento de todos 
mais autônomo e participativo (Simonetti 2017; Lima 2016; Shapiro 2003; Rahde 2002).

O indivíduo se utiliza da iconografia, baseado em parte na semelhança entre o objeto-significado e o objeto- pivô tal como um convite interativo com autor-leitor (Barbosa \& Moura 2013).

"ACO" e "NACO" são personagens fictícios criados para serem agentes facilitadores na aprendizagem da equipe de enfermagem, reconhecendo-os como instrumentos pedagógicos lúdicos importantes para reflexão da equipe de enfermagem na administração dos anticoagulantes orais (Rahde 2002).

A criação de personagem é uma ação significativa e criativa quando o autor através dela consegue abordar os problemas gerais do seu tempo, mesmo os mais abstratos, como problemas individualmente seus, e com vital importância.

Torna-se interessante e popular quando ocorre o equilíbrio entre os modelos universais, facilmente reconhecíveis pelos leitores e os argumentos originais, no sentido de despertar o interesse em conhecer algo novo (Barbosa \& Moura 2013; Shapiro 2003).

O uso dos personagens ilustrou aspectos dos sujeitos no cotidiano do serviço, oportunizando o estabelecimento das relações entre ficção e a realidade dos processos de trabalho; elemento determinante para a efetividade da educação permanente dos profissionais, em âmbito local (Shapiro 2003; Rahde 2002).

No estudo foi possível identificar como fator dificultador o número reduzido de artigos encontrados sobre o tema. Dessa forma, pode-se perceber a incipiência dos estudos científicos publicados caracterizando que se trata de uma temática carente de maior desenvolvimento. O desconhecimento e a falta de incentivo para adoção de estratégias de proteção à propriedade intelectual assim como fato que na cultura hospitalar mesmo diante do uso de novas metodologias para a educação do trabalhador é possível perceber um despreparo para ações inovativas nas capacitações.

A necessidade do desenvolvimento de uma postura empreendedora na enfermagem para que as tecnologias e inovações de propriedade intelectual dos enfermeiros sejam registradas e protegidas adequadamente.

O Programa de Pós-Graduação em Saúde e Tecnologia no Espaço Hospitalar é uma importante ferramenta disseminadora dos conceitos de inovação tecnológica, efetividade, sistema de propriedade intelectual. A criação dos personagens e proteção à propriedade intelectual destes é resultado do diálogo entre a Universidade/Hospital/Experiência profissional, buscando atender tanto a capacitação dos profissionais, o cuidado e proteção aos usuários e ressalta a potência intelectual do profissional enfermeiro como agente transformador de sua prática.

\section{Considerações Finais}

O hospital é um ambiente complexo no qual o enfermeiro exerce múltiplas funções e jornadas diversas no processo do cuidar. É exigida por parte da equipe de enfermagem a busca da superação de lacunas que surgem em suas práticas. O uso de personagens aparece como uma maneira criativa e atrativa de aproximar a equipe do conhecimento sobre o cuidado prestado ao paciente hospitalizado em uso de anticoagulantes orais. O registro itede obra intelectual é uma importante iniciativa que apresenta a capacidade de originalidade e inventividade do Enfermeiro. Devido à complexidade do assunto este estudo propõe novas pesquisas relacionadas com a temática, já que este não pretende produzir conhecimento absoluto, mas busca contribuir com processos de criações lúdicas para a educação em saúde em Enfermagem.

\section{Referências}

Barbosa, E. F., \& Moura, D. G. (2013). Boletim técnico SENAC: Metodologias ativas de aprendizagem na Educação Profissional e Tecnológica. (2. Ed.), 39: Senac.

Barbosa, L. M., Silva, M. S. A., Cavalcanti, A. M. F., Nogueira, L. D. de M., Francisco, M. A., Santos, M. P. de M., Lima, C. H. R., Negreiros, J. H. C. N., Monteiro, J. L. G. C., \& Laureano Filho, J. R. Protocol for surgical treatment of patients using anticoagulants and antiplatelet agents. Research, Society and Development, 9(9), e670997726, 2020. 10.33448/rsd-v9i9.7726. https://rsdjournal.org/index.php/rsd/article/view/7726. 
Research, Society and Development, v. 10, n. 3, e27110313250, 2021

(CC BY 4.0) | ISSN 2525-3409 | DOI: http://dx.doi.org/10.33448/rsd-v10i3.13250

Costa D. G., Pasin S. S., Magalhães A. M. M., Moura G. M. S. S., Rosso C. B., \& Saurin T. A. (2018). Análise do preparo e administração de medicamentos no contexto hospitalar com base no pensamento Lean. Escola Anna Nery, 22(4). https://www.scielo.br/pdf/ean/v22n4/pt_1414-8145-ean-22-04e20170402.pdf. 10.1590/2177-9465-EAN-2017-0402

Fakih, F. T., Freitas, G. F., \& Secoli, S. R. (2009). Medicação: aspectos ético-legais no âmbito da enfermagem. Revista Brasileira de Enfermagem. São Paulo, 1(62). (pp. 132-135). http://www.scielo.br/pdf/reben/v62n1/20.pdf.

Field, Syd. (1995). Manual do Roteiro: Objetiva.

Freitas D., Ribeiro, K., Oliveira, J. L. C., Matos F. G. O. A., Carvalho, A. R. S., \& Ross, C. (2017). Diagnósticos de Enfermagem entre usuários de anticoagulante oral acompanhado em Ambulatório. Revista Bahiana de Enfermagem, 31(3). 1-11. https://portalseer.ufba.br/index.php/enfermagem/article/viewFile/20356/15074. 10.18471/rbe.v31i3.20356

Lima, V. V. (2016). Espiral construtivista: uma metodologia ativa de ensino-aprendizagem. Interface - Comunicação, Saúde, Educação. 21(61). 421-434. http://dx.doi.org/10.1590/1807-57622016.0316.

Mendes, K. D., Silveira, R. C. C. P., \& Galvão, C, M. (2008). Revisão integrativa: método de pesquisa para a incorporação de evidências na saúde e na enfermagem. Texto \& Contexto - Enfermagem. 17(4), 758-764. http://dx.doi.org/10.1590/s0104-07072008000400018.

Polit, D. F., \& Beck, C. T. (2018). Fundamentos de pesquisa em enfermagem: avaliação de evidências para a prática da enfermagem. (9a ed.): Artmed.

Rahde, M. B. F. (2002). Iconografia e comunicação: a construção de imagens míticas. Revista Logos. 09(17). 07-18 http://www.logos.uerj.br/PDFS/anteriores/logos17.pdf\#page=8.

Rocha, B. S. U., Giordani, A. T., Fujita, L. A. S., \& Reinaldi, M. A. de A. Main challenges in Nursing teaching: a systematic review of literature. Research, Society and Development, 9(5), p. e87951487. 10.33448/rsd-v9i5.1487. https://rsdjournal.org/index.php/rsd/article/view/1487.

Serrano Júnior, C. V., Soeiro, A. M., Leal, T. C. A. T., Godoy, L. C., Biselli, B., Hata, L. A., Martins, E. B., Abud-Manta, I. C. K., Tavares, Caio A. M., Cardozo, F. A. M., \& Oliveira Júnior, M. T. (2019) Posicionamento sobre Antiagregantes Plaquetários e Anticoagulantes em Cardiologia - 2019. Arquivos Brasileiros de Cardiologia. (pp. 111-134). http://dx.doi.org/10.5935/abc.20190128.

Shapiro, S. S. (2003). Treating Thrombosis in the 21st Century. New England Journal Of Medicine. 349(18). (pp. 1762-1764). Massachusetts Medical Society. http://dx.doi.org/10.1056/nejme038152.

Simonetti, S. H., Massa, V. C., \& França, J. Í. D. (2017). Método Educativo Convencional e Inovador para o aprendizado do usuário de anticoagulação oral Enfermagem em Foco. 04(08). (pp. 03-06). http://revista.cofen.gov.br/index.php/enfermagem/article/view/938/407. 